\title{
Mediolateral damping of an overhead body weight support system assists stability during treadmill walking
}

\author{
M. Bannwart ${ }^{1,2^{*}}$ D, S. L. Bayer ${ }^{1}$, N. König Ignasiak³ ${ }^{3}$ M. Bolliger ${ }^{1}$, G. Rauter ${ }^{1,2,4}$ and C. A. Easthope ${ }^{1,5}$
}

\begin{abstract}
Background: Body weight support systems with three or more degrees of freedom (3-DoF) are permissive and safe environments that provide unloading and allow unrestricted movement in any direction. This enables training of walking and balance control at an early stage in rehabilitation. Transparent systems generate a support force vector that is near vertical at all positions in the workspace to only minimally interfere with natural movement patterns. Patients with impaired balance, however, may benefit from additional mediolateral support that can be adjusted according to their capacity. An elegant solution for providing balance support might be by rendering viscous damping along the mediolateral axis via the software controller. Before use with patients, we evaluated if controlrendered mediolateral damping evokes the desired stability enhancement in able-bodied individuals.

Methods: A transparent, cable-driven robotic body weight support system (FLOAT) was used to provide transparent body weight support with and without mediolateral damping to 21 able-bodied volunteers while walking at preferred gait velocity on a treadmill. Stability metrics reflecting resistance to small and large perturbations were derived from walking kinematics and compared between conditions and to free walking.

Results: Compared to free walking, the application of body weight support per-se resulted in gait alterations typically associated with body weight support, namely increased step length and swing phase. Frontal plane dynamic stability, measured by kinematic variability and nonlinear dynamics of the center of mass, was increased under body weight support, indicating reduced balance requirements in both damped and undamped support conditions. Adding damping to the body weight support resulted in a greater increase of frontal plane stability.

Conclusion: Adding mediolateral damping to 3-DoF body weight support systems is an effective method of increasing frontal plane stability during walking in able-bodied participants. Building on these results, adjustable mediolateral damping could enable therapists to select combinations of unloading and stability specifically for each patient and to adapt this in a task specific manner. This could extend the impact of transparent 3-DoF body weight support systems, enabling training of gait and active balance from an early time point onwards in the rehabilitation process for a wide range of mobility activities of daily life.
\end{abstract}

Keywords: Body weight support, Mediolateral stability, Balance, Gait, Rehabilitation

\footnotetext{
* Correspondence: mathias.bannwart@hest.ethz.ch

'Spinal Cord Injury Center, Balgrist University Hospital, University of Zurich, Zurich, Switzerland

${ }^{2}$ Sensory Motor Systems Laboratory, Department of Health Sciences and Technology, Swiss Federal Institute of Technology, Zurich, Switzerland

Full list of author information is available at the end of the article
}

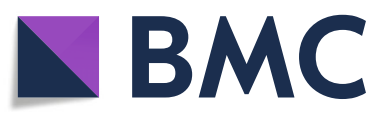

(C) The Author(s). 2020 Open Access This article is licensed under a Creative Commons Attribution 4.0 International License, which permits use, sharing, adaptation, distribution and reproduction in any medium or format, as long as you give appropriate credit to the original author(s) and the source, provide a link to the Creative Commons licence, and indicate if changes were made. The images or other third party material in this article are included in the article's Creative Commons. licence, unless indicated otherwise in a credit line to the material. If material is not included in the article's Creative Commons licence and your intended use is not permitted by statutory regulation or exceeds the permitted use, you will need to obtain permission directly from the copyright holder. To view a copy of this licence, visit http://creativecommons.org/licenses/by/4.0/ The Creative Commons Public Domain Dedication waiver (http://creativecommons.org/publicdomain/zero/1.0/) applies to the data made available in this article, unless otherwise stated in a credit line to the data. 


\section{Background}

In the past 3 decades, neurological injuries have been the leading cause of disease burden and second leading cause of deaths worldwide [1]. Around $60 \%$ of the affected patients manifest gait impairments [2], which contribute strongly to the disease burden. Locomotor rehabilitation programs aim at rehabilitating patients' walking capacity and reducing gait impairments. Patients not able to safely bear full body weight struggle without large assistance and are prone to adopt compensatory movement strategies. Body weight support (BWS) is a promising path to retrain physiological walking and avoid the development of compensatory patterns. Testimony to this are the numerous devices on the market and currently in development and the rising adoption in rehabilitation taxonomy [3]. BWS is generally provided through a harness which applies vertical (VT) forces to the pelvis or trunk to achieve partial gravity reduction. Together with fall prevention mechanisms, this creates a safe and permissible environment that enables and facilitates early locomotor training as well as training of many mobility-related skills. Compared to handheld assistive devices such as a walking frame, BWS systems provide specific benefits for locomotor trainings: 1) BWS maximizes controlled weight bearing on the legs which enhances lower limb electromyographic activity and interlimb coordination through appropriate sensory input and walking posture [4]. 2) BWS enables patients to swing their arms naturally, thus supporting forward motion and balance, the ability to control body posture dynamics that prevent falling [5], on a mechanical level [6-9]. 3) Natural arm swing induces rhythmic activation of the shoulder flexors and extensors which reinforces the patterned output of spinal locomotor networks, herewith supporting positive neuroplasticity [10-15]. 4) BWS prevents compensatory balance strategies that use the arms and thus results in maximized motor learning [16].

During the last years, BWS systems have become more elaborate and moved away from stationary, treadmillcoupled systems to highly transparent systems which allow both treadmill and overground walking. Transparent systems are defined by the ability to not apply any assistance/resistance to free motion [17]. This is especially relevant in evoking physiological gait patterns that allow seamless translation to a non-supported environment. Specifically, the attachment point must replicate the patient's motion in sagittal and frontal planes with minimal delay. The basic construction principles behind BWS systems can be grouped into frame-based systems that are either stationary (e.g. Lokomat [18], G-EO [19], KineAssist MX [20]) or mobile (Biodex [21], Andago [22]). A second group of devices are ceiling mounted systems that are either based on a single rail mounting giving two degrees of freedom (2-DoF: Zero-G [23], Vector, Safe Gait) or multiple rail mounting providing three degrees of freedom (3-DoF: FLOAT [24], Rysen [25]). In contrast to frame-based systems and 2-DoF ceilingmounted systems, 3-DoF BWS systems enable greater freedom of movement in all directions. The increased DoF in the most recent BWS systems allow physiological training of various activities with only minor deviations $[3,26-28]$ and might be an effective way to train balance control. Particularly for patients with neurological disorders, who fall twice as frequently as age-matched controls [29], such versatile balance training could improve functional recovery [30] and prevent fall-related loss of mobility and quality of life.

Increasing a BWS system's DoF inherently increases the demands on patients' balance capacity. If the challenge becomes too large, patients are prone to resort to crutches or walkers for external stabilization ultimately resulting in compensatory movement strategies. Preventing compensation and instead challenging balance control in a patient-tailored manner would be desirable [31]. An elegant solution which does not require reliance on external, arm-based support, would be a stabilizing mode as an integrated feature of the BWS system. Such a feature must be easily scalable to the functional level of the patient to provide a sufficiently large challenge for highly functioning patients, while avoiding overly challenging conditions for less functioning patients [32, 33].

A stabilizing feature of BWS systems could be especially helpful in the frontal plane. Maintenance of frontal plane balance during walking has been shown to require active control of lateral foot placement, which is highly dependent on successful integration of sensory feedback. In the sagittal plane, humans can use passive dynamic limb properties for stabilization. This reduces the relevance of active control of foot placement along the anteroposterior (AP) axis and therewith also the demands on the integration of sensory feedback [34]. One way of scaling the level of frontal plane support to each patient's capabilities is to provide mediolateral (ML) stabilizing forces to the body in the frontal plane. Such ML forces can limit excessive center of mass (CoM) excursion in regard to the base of support (BoS), the minimum area enclosing the body's contact with the ground [35], thus reducing balance demands. When describing BWS systems, we describe the position and forces acting on the end-effector which is considered as the attachment point of the BWS system to the BWS harness. Due to their architecture, single rail BWS systems inherently engender pendulum forces. When patients deviate too far from the midline, these forces move the BWS endeffector back into a position directly under the rail. While walking, patients can adapt their step width and 
cadence to use these forces for passive stabilization in the frontal plane [36-39]. Pendulum forces, however, cannot be adjusted to patients' capacities or removed when patients become self-reliant with time. This limits their usefulness for adaptive stabilization and active balance training in the frontal plane. A different way to increase stability when walking in a straight line is by using lateral spring elements. This has been reported effective in young and elderly [40-43], as well as in patients with different central nervous system disorders [44, 45]. Employing lateral springs reduces energy and control costs, inferring that gait complexity and challenge may be decreased [40-42]. Compared to the pendulum forces of single rail systems, spring elements can be adjusted to produce larger or smaller stabilization forces. The spring-elements, however, are cumbersome requiring additional hardware and attachment points and are not location independent, so are only useful in a treadmill environment. A third option to influence gait stability are ML damping forces. These forces have the benefit that they can be adapted on the fly and can be applied independently of the user's position and orientation in space. This will be especially valuable for 3-DoF overground BWS systems. However, damping forces oppose all movements along the defined axis, inferring that while unwanted COM displacements are reduced, desired displacements require increased effort. Hence, it remains to be investigated if damping of the BWS endeffector ultimately stabilizes ML COM motion and therewith proves beneficial for patients with balance impairments.

We investigated in this study how ML damping of the BWS end-effector affects frontal plane stability in able-bodied participants during walking. Stability during walking is commonly investigated using dynamic stability, the postural control process in which both the COM and the BoS are in motion [35]. It can be further divided into global or local dynamic stability, which are a system's ability to resist large respectively small perturbations. In contrast to large external perturbations, small perturbations are naturally occurring fluctuations which arise from neuromotor noise or other internal perturbations [46]. In this study, we examined both global and local stability under transparent BWS and ML damped modes. This was compared to free walking. Our investigation aims at providing novel insight into how human-robot interaction during unloaded walking can subtly improve or challenge balance control without fundamentally distorting basic movement patterns. We hypothesized that mechanically damping the ML motion of the end-effector increases global and local frontal plane stability and therewith reduces balance demands. If this proves to be the case, ML damping can be applied in future studies with patients with balance impairments to test its effectiveness in a clinical setting.

\section{Methods \\ Participants}

Twenty-one young, able-bodied participants gave their informed written consent to participate in this study that was approved by the local ethics committee (KEK: 2016-01093) and conducted according to the Declaration of Helsinki and Good Clinical Practice. Inclusion criteria were that participants were older than 18 years of age and weighed less than $120 \mathrm{~kg}$. The participants (11 female) were on average $26.8 \pm$ 3.5 years old, $1.74 \pm 0.1 \mathrm{~m}$ tall, weighed $65.7 \pm 12.5 \mathrm{~kg}$ (mean \pm 1 standard deviation, and walked on average with a self-selected velocity of $0.81 \pm 0.14 \mathrm{~m}$ per second during the experiment).

\section{Experimental protocol}

Participants wore tight-fitting attire and were equipped with a specially modified BWS harness that allowed accurate placement of 19 spherical, retro-reflective markers with $14 \mathrm{~mm}$ diameter at bony landmarks (Fig. 1). Demographic information such as age, height, and weight were noted.

Afterwards, the participants walked for $5 \mathrm{~min}$ on a treadmill (Zebris FDM-T, Isny, Germany) wearing the harness but without BWS for familiarization purposes [47]. During this time, the participants were instructed to self-regulate their speed (increments of $0.028 \mathrm{~m} / \mathrm{s}$, no visual feedback) to achieve a "comfortable, everyday walking pace" which they would be able keep up for a total of 30-40 min. The median speed of minutes 3-5 was recorded as preferred gait velocity and was applied for the remaining experiment.

Following this familiarization, three conditions were applied in counterbalanced (equal distribution of the six possible orders among all subjects), pseudorandomized order: 30\% BWS with damped ML motion (damped), $30 \%$ BWS without damping which enables fully transparent motion in the frontal plane (transparent) and typical walking without BWS but while wearing the harness (free).

In each experimental condition, participants walked for $10 \mathrm{~min}$. The first $2 \mathrm{~min}$ were chosen to allow subjects to familiarize themselves with the condition. The remaining $8 \mathrm{~min}$ provided the necessary recording time for robust calculation of the parameters we used to quantify changes in stability $[48,49]$. Between individual conditions, subjects were always detached from the BWS system and received a 5-min break during which they could rest in a standing position or lean on the treadmill's handrails. 


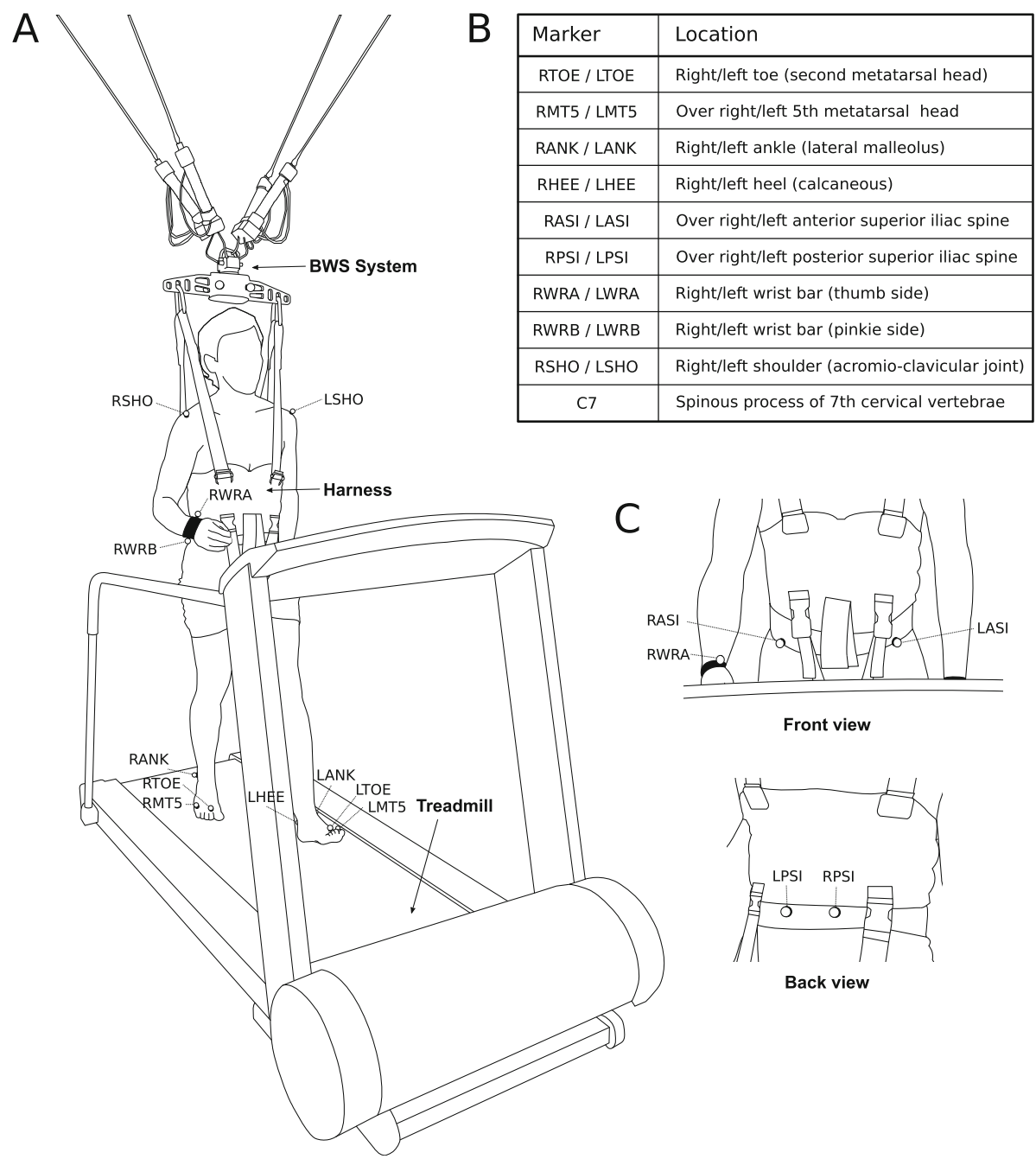

Fig. 1 Experimental setup. a Subject walking with a harness and assistance from a 3-DoF, robotic BWS system (the FLOAT) on the treadmill. b List of used markers and their anatomical locations. C) Front and back view of pelvis marker placement next to BWS harness

\section{Equipment}

BWS was provided through the FLOAT, a cable-driven parallel robot, which allows large freedom of movement in an architecture-dependent workspace volume $(2.35 \mathrm{~m}$ wide $\times 7.82 \mathrm{~m}$ long $\times 3.5 \mathrm{~m}$ high for our gait lab). Apart from safety (fall prevention) and up to $60 \%$ BWS, this active 3-DoF BWS system can provide damping along the AP or ML end-effector axes. We previously quantified the FLOAT's transparency in a preceding experiment with a calibrated test rig that simulated walking of able-bodied participants and slow walking patients. These measurements showed that during transparent BWS mode only small interaction forces in the range of 3-18 $\mathrm{N}$ can be perceived in the horizontal plane during constant motion [50]. For the transparent and damped conditions, 30\% BWS was applied. This reflects experience from clinical practice, where therapists frequently selected this level of assistance for early BWS training in patients that required substantial assistance. In the damped condition, ML stabilizing forces were rendered additionally to the $30 \%$ BWS. These were generated by continuously applying velocity-dependent damping to the ML end-effector axis:

$$
F_{\text {damping }}=-c \frac{d x}{d t}=-c v
$$

c: viscous damping coefficient, v: mediolateral velocity of the BWS end-effector. The direction of the damping force was opposed to the end-effector mediolateral motion and a viscous damping coefficient of $120 \mathrm{~N} \mathrm{~s} / \mathrm{m}$ (corresponding to the strongest damping setting available in the FLOAT) was used across all subjects with a maximal force magnitude saturated at $200 \mathrm{~N}$. During conditions, marker positions were recorded at $200 \mathrm{~Hz}$ using 10 passive infrared cameras (T10/T20, Vicon, 
Oxford, UK). The FLOAT's end-effector position was also recorded via optical motion tracking to ensure that the damping worked as intended.

\section{Outcome parameters}

To investigate effects of BWS and damping on gait and dynamic stability, we selected 11 outcome parameters (Table 1). Two of these were spatiotemporal parameters along the AP axis: duty cycle and step length. These parameters were selected to verify that damping did not affect the spatiotemporal pattern along the AP direction. Five of these parameters quantified global dynamic stability along the ML axis: step width, ML COM sway, ML margins of stability (MoS), and the coefficients of variation $(\mathrm{CoV})$ of step width and ML COM sway. ML MoS, which describes the positional relationship of COM and BOS at midstance, and the related measures step width and ML COM sway are direct measures of global dynamic stability. For the calculation of all COM-related parameters, we used an approximated COM (aCOM) calculated as the intersection of two vectors crossing the pelvis from left posterior spina iliac - right anterior spina iliac and left anterior spina iliac - right posterior spina iliac [51]. This was necessary because the BWS harness prevented reliable placement of upper trunk markers. Into the parameters describing global dynamic stability, we also included kinematic variability calculated from step width and ML aCOM sway. Many previous studies have shown that kinematic variability can be sensitive to changes in stability, e.g. between young and old [52] or fallers vs. non-fallers [53]. Changes in variability are however indirect measures of global dynamic stability and cannot provide causality because they are influenced by many other factors [54]. As descriptors of local dynamic stability, four nonlinear stability parameters based on the short-term maximum Lyapunov exponent $\left(\lambda_{s}\right)$ were included. The $\lambda_{s}$ were calculated from ML and VT aCOM position $\left(\lambda_{\mathrm{s} \text { pos }}\right)$ and velocity $\left(\lambda_{\mathrm{s} \text { vel }}\right) . \lambda_{\mathrm{s}}$ takes the effect of the preceding step(s) on the next step into account while kinematic variability and direct measures of global dynamic stability treat each gait cycle as independent from others. $\lambda_{\mathrm{s}}$ is therefore especially suitable for detecting differences between position-timing dependent systems such as COM motion while walking [55-57]. $\lambda_{\mathrm{s}}$ can be used as a proxy measure of freedom in ML COM motion [58]. A higher $\lambda_{\mathrm{s}}$ indicates larger chaotic elements of a system, while $\lambda_{\mathrm{s}}$ close to zero indicates a stable system [59]. $\lambda_{\mathrm{s}}$ was chosen due to its

Table 1 Outcome parameters

\begin{tabular}{|c|c|c|c|}
\hline Name & Definition & Unit & $\begin{array}{l}\text { Primary effect } \\
\text { axis }\end{array}$ \\
\hline \multicolumn{4}{|l|}{ AP spatiotemporal parameters } \\
\hline Duty cycle & Percentage of stance phase over the whole cycle duration & $\%$ & anteroposterior \\
\hline Step length & $\begin{array}{l}\text { Anteroposterior distance between contralateral heel markers at their respective heel strikes } \\
\text { plus the distance the stance foot moved back with the treadmill belt during this time }\end{array}$ & $\mathrm{m}$ & anteroposterior \\
\hline \multicolumn{4}{|c|}{ Direct measures of global dynamic stability } \\
\hline Step width & Mediolateral distance between contralateral heel markers at their respective heel strikes & $\mathrm{m}$ & mediolateral \\
\hline $\begin{array}{l}\text { ML approximated COM } \\
\text { (aCOM) sway }\end{array}$ & Difference between the ML extrema of the aCOM within each step & $\mathrm{m}$ & mediolateral \\
\hline $\begin{array}{l}\text { ML Margins of Stability } \\
\text { (ML MoS) }\end{array}$ & $\begin{array}{l}\text { Shortest distance of the floor-projected aCOM during midstance to the nearest edge of the } \\
\text { base of support } \\
\text { BoS was defined as the smallest convex hull spanning all points (heel, ankle, 5th metatarsal } \\
\text { and 2nd metatarsal) in contact with the ground }\end{array}$ & $\mathrm{m}$ & mediolateral \\
\hline \multicolumn{4}{|c|}{ Indirect measures of global dynamic stability (kinematic variability) } \\
\hline Step width (CoV) & Standard deviation divided by the mean of step width & $\mathrm{m}$ & mediolateral \\
\hline $\begin{array}{l}\text { ML approximated COM } \\
\text { (aCOM) sway (CoV) }\end{array}$ & Standard deviation divided by the mean of ML aCOM sway & $\mathrm{m}$ & mediolateral \\
\hline \multicolumn{4}{|c|}{ Measures of local dynamic stability (nonlinear parameters) } \\
\hline $\mathrm{ML} \lambda_{\mathrm{s} \text { pos }}$ & Short-term maximum Lyapunov exponent from time series of ML aCOM position & $\begin{array}{l}\text { arbitrary } \\
\text { unit }\end{array}$ & mediolateral \\
\hline$M L \lambda_{s}$ vel & Short-term maximum Lyapunov exponent from time series of ML aCOM velocity & $\begin{array}{l}\text { arbitrary } \\
\text { unit }\end{array}$ & mediolateral \\
\hline $\mathrm{VT} \lambda_{\mathrm{s} \text { pos }}$ & Short-term maximum Lyapunov exponent from time series of $\mathrm{VT}$ aCOM positions & $\begin{array}{l}\text { arbitrary } \\
\text { unit }\end{array}$ & vertical \\
\hline $\mathrm{VT} \lambda_{\mathrm{s} \text { vel }}$ & Short-term maximum Lyapunov exponent from time series of VT aCOM velocity & $\begin{array}{l}\text { arbitrary } \\
\text { unit }\end{array}$ & vertical \\
\hline
\end{tabular}


validated representation of local dynamic stability which is not true for long-term Lyapunov exponents [60].

\section{Data processing}

For the calculation of the 11 selected parameters, the recorded motion data from minutes $2-10$ of each condition was reconstructed (Nexus 2.7, Vicon, Oxford, UK) and labelled. From now on onwards, we term such processed, individual data sets "trials". Custom MATLAB (R2018a, the MathWorks, Natick, USA) scripts were used for all further analysis. Gait events (heel strike, toe off) were algorithmically set for each trial based on velocity sign changes of the heel and toe markers according to Zeni et al. and visually verified [61]. For all parameters apart from $\lambda_{\mathrm{s}}$, marker data was segmented into gait cycles and linearly interpolated to 500 data points. Step length, step width, duty cycle, ML MoS, and ML aCOM sway were then extracted for each individual gait cycle and subsequently averaged per trial for each subject. The CoV for step width and ML aCOM sway was determined from the averaged gait cycle values and standard deviations obtained for each individual trial.

For the investigation of local dynamic stability using $\lambda_{s}$, it is necessary to first reconstruct a higher dimension state-space from each dimension of the aCOM data [62]. For this, aCOM position and velocity traces in ML and VT directions were each downsampled to $50 \mathrm{~Hz}$ to remove contaminating artefacts. Trials were cropped to the same length, and the final $15 \mathrm{~s}$ of each trial were discarded to avoid transients (slowing down or stopping). We then reconstructed higher dimensional dynamics according to Takens' Theorem [63] by embedding the original timeseries with time-delayed surrogate copies [62, $64,65]$ as singularly defined by two parameters: a time delay $(\tau)$ and the number of embedding dimensions (D). These were determined separately for each trial: the minimal number of embedding dimensions was identified by observing the behavior of the closest geometrical neighbors using the false nearest neighbor method [66]. Similarly, the time delay between embedding dimensions was identified as the first local minima of the average mutual information function between the original time series and it's lagged copy [67]. To enable comparison of $\lambda_{s}$ between trials, we defined a common state space using the median number of embedding dimensions $(\mathrm{D}=5)$ and median time delay $(\tau=22 \mathrm{~ms})$. The algorithm developed by Wolf was then used to determine $\lambda_{s}$ for each trial [68].

\section{Statistical analysis}

Statistical comparisons of all parameters were performed in SPSS (v25, IBM Corp., Armonk, USA). To validate if damping was effective, we first compared the ML and VT end-effector axes between transparent and damped conditions using a repeated measures t-test. Normality of data was tested with a Shapiro-Wilk test and the Holm-Bonferroni correction was used to counteract the problem of multiple comparisons. A one-way repeated measures multivariate analysis of variance (RM-MANOVA) was then used to assess if the three experimental conditions had significant different effects on the combination of the selected 11 outcome parameters: duty cycle, step width, step width variability, step length, aCOM sway, aCOM sway variability, MoS, ML $\lambda_{s}$ pos, ML $\lambda_{\text {s vel, }}$ VT $\lambda_{\text {s pos, }}$ and VT $\lambda_{\text {s vel. }}$ RM-MANOVA assumptions were checked beforehand including independence of observations, adequate sample size, absence of univariate and multivariate outliers, multivariate normality (using "MVN: a web-tool for assessing multivariate normality" [69]), linear relationship between each pair of dependent variables, and absence of multicollinearity. Pillai's trace was selected as the test statistic of the RM-MANOVA due to its robustness against small violations of assumptions [70]. A significant RMMANOVA was followed up with univariate, repeated measures analyses of variance for each outcome parameter to test which of the parameters were affected. Any violation of sphericity as detected by Mauchly's test of sphericity was corrected using the Greenhouse-Geisser correction. Group differences were mapped to the individual conditions (free vs. damped vs. transparent) using pairwise multiple comparisons tests between all conditions corrected according to Bonferroni-Sidak. A significance level of alpha $=0.05$ was set for all comparisons.

\section{Results}

The effectiveness of damping the ML end-effector axis was first verified by comparing the ML end-effector excursion during the damped against the transparent condition (Fig. 2). ML end-effector excursion was clearly reduced with damping ( $\mathrm{t}(20)=13.19, p<0.001$ ) while the VT excursion showed no significant difference $(\mathrm{t}$ $(20)=2.19, p=0.081)$.

The RM-MANOVA showed a significant effect of the stability conditions on the measured parameters $(\mathrm{V}=$ 1.72 , $\mathrm{F}(22,62)=17.04, p<0.001)$. Separate univariate tests (individual analyses of variance) revealed that only the MoS were not affected by the differences between experimental conditions (Table 2).

Pairwise multiple comparisons for the statistically significant parameters allowed us to distinguish three parameter groups with different response patterns:

1) Spatiotemporal and local dynamic stability parameters along the VT axis showed differences only between the free condition and the transparent/damped conditions (Fig. 3). These parameters, whose response we allocated mainly to 


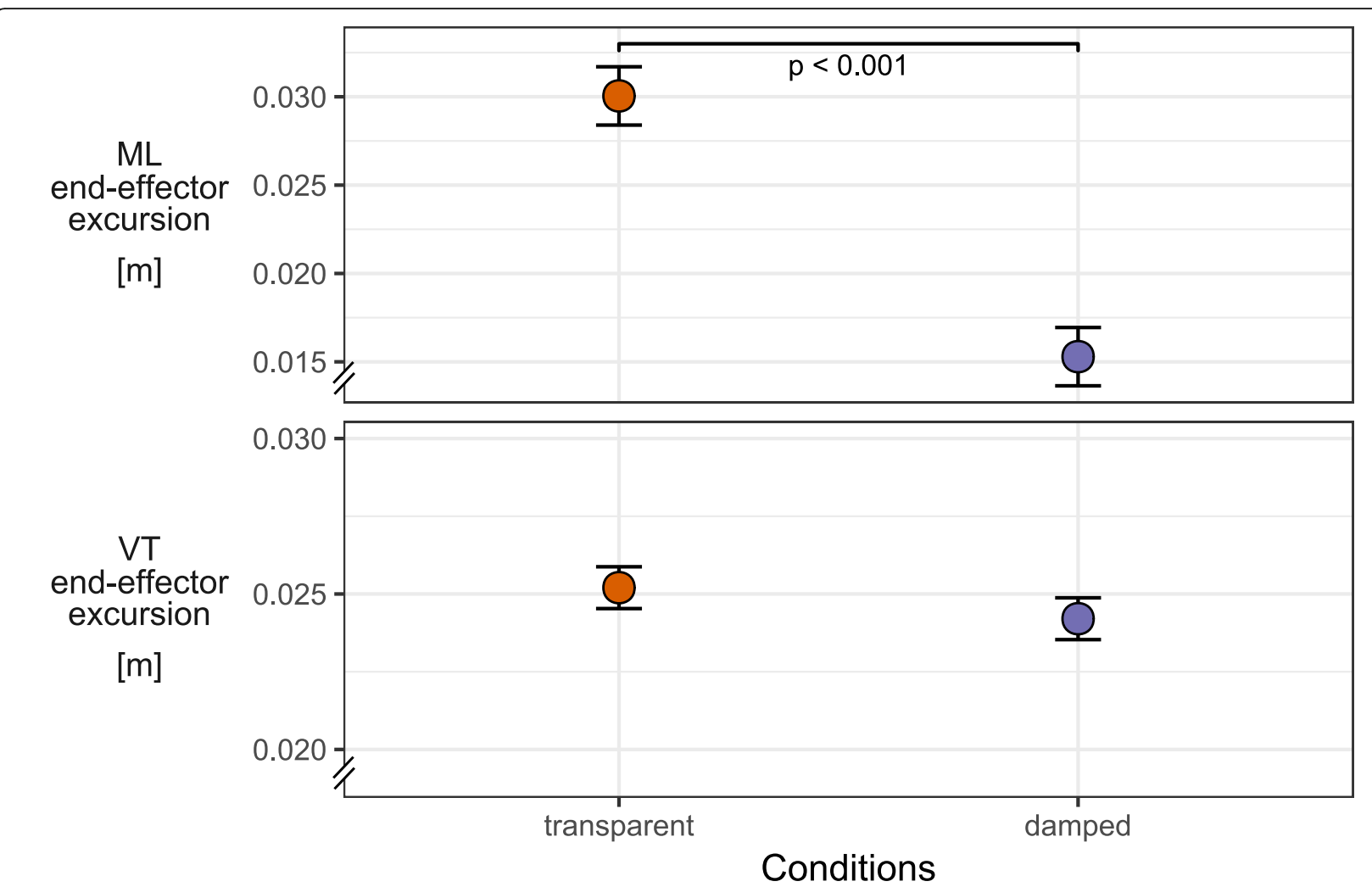

Fig. 2 Effects of damping on BWS end-effector motion. Line plots of estimated marginal means over the transparent and damped conditions for mediolateral and vertical BWS end-effector excursion. Plot includes error bars specifying 95\%-confidence intervals (within-subject, [71]) and statistically significant differences denoted by $p$-values. Abbreviations: $\mathrm{ML}$ - mediolateral, $\mathrm{VT}$ - vertical

effects in the AP and VT axis, included duty cycle, step length and $\lambda_{\mathrm{s}}$ along the VT axis $\left(\lambda_{\mathrm{s} \text { pos }}, \lambda_{\mathrm{s} \text { vel }}\right)$. The presence of BWS was the only separating experimental factor. While step length increased with the use of BWS, the remaining three parameters showed a clear decrease. Reduced VT $\lambda_{\mathrm{s}}$ pos and VT $\lambda_{\mathrm{s} \text { vel }}$ indicate increased local dynamic stability in the VT axis. A reduced duty cycle

Table 2 Repeated measures ANOVA test statistics for outcome parameters

\begin{tabular}{lll}
\hline Parameter & F-statistic & $\boldsymbol{P}$-value \\
\hline step length & $\mathrm{F}(1.41,28.23)=3.95)$ & $p=0.044$ \\
duty cycle & $\mathrm{F}(1.36,27.21)=37.88 .15$ & $p<0.001$ \\
step width & $\mathrm{F}(1.45,29.06)=4.73$ & $p=0.014$ \\
ML aCOM sway & $\mathrm{F}(1.37,27.46)=65.93$ & $p<0.001$ \\
ML MoS & $\mathrm{F}(1.55,31.00)=1.59$ & $p=0.22$ \\
step width variability & $\mathrm{F}(1.41,28.24)=42.24$ & $p<0.001$ \\
aCoM sway variability & $\mathrm{F}(1.40,28.04)=49.81$ & $p<0.001$ \\
ML $\lambda_{\text {s pos }}$ & $\mathrm{F}(2,40)=27.58$ & $p<0.001$ \\
ML $\lambda_{\text {s vel }}$ & $F(2,40)=56.86$ & $p<0.001$ \\
VT $\lambda_{\text {s pos }}$ & $F(1.43,28.53)=34.14$ & $p<0.001$ \\
VT $\lambda_{\text {s vel }}$ & $F(1.45,29.03)=41.86$ & $p<0.001$ \\
\hline
\end{tabular}

indicates a shift in relative duration from stance to swing.

2) For direct global dynamic stability parameters, the experimental conditions induced no coherent pattern and large variability was present for some of these parameters (Fig. 4). This included step width, ML aCOM sway, and MoS. ML aCOM sway was minimal during the transparent condition, increased in the damped condition, and further increased in the free condition. Step width was only decreased during the transparent condition and remained comparable between free and damped conditions. The MoS were not significantly affected by the experimental conditions.

3) Kinematic variability and local dynamic stability parameters along the ML axis showed a stepwise change from the free to transparent to damped condition (Fig. 5). This category included step width variability, ML aCOM sway variability, ML $\lambda_{s}$ pos, and ML $\lambda_{\mathrm{s} \text { vel. }}$. All parameters showed a significant decrease from free to damped conditions. Between the transparent and damped conditions this decrease was less pronounced and was only not significant for step width variability. 


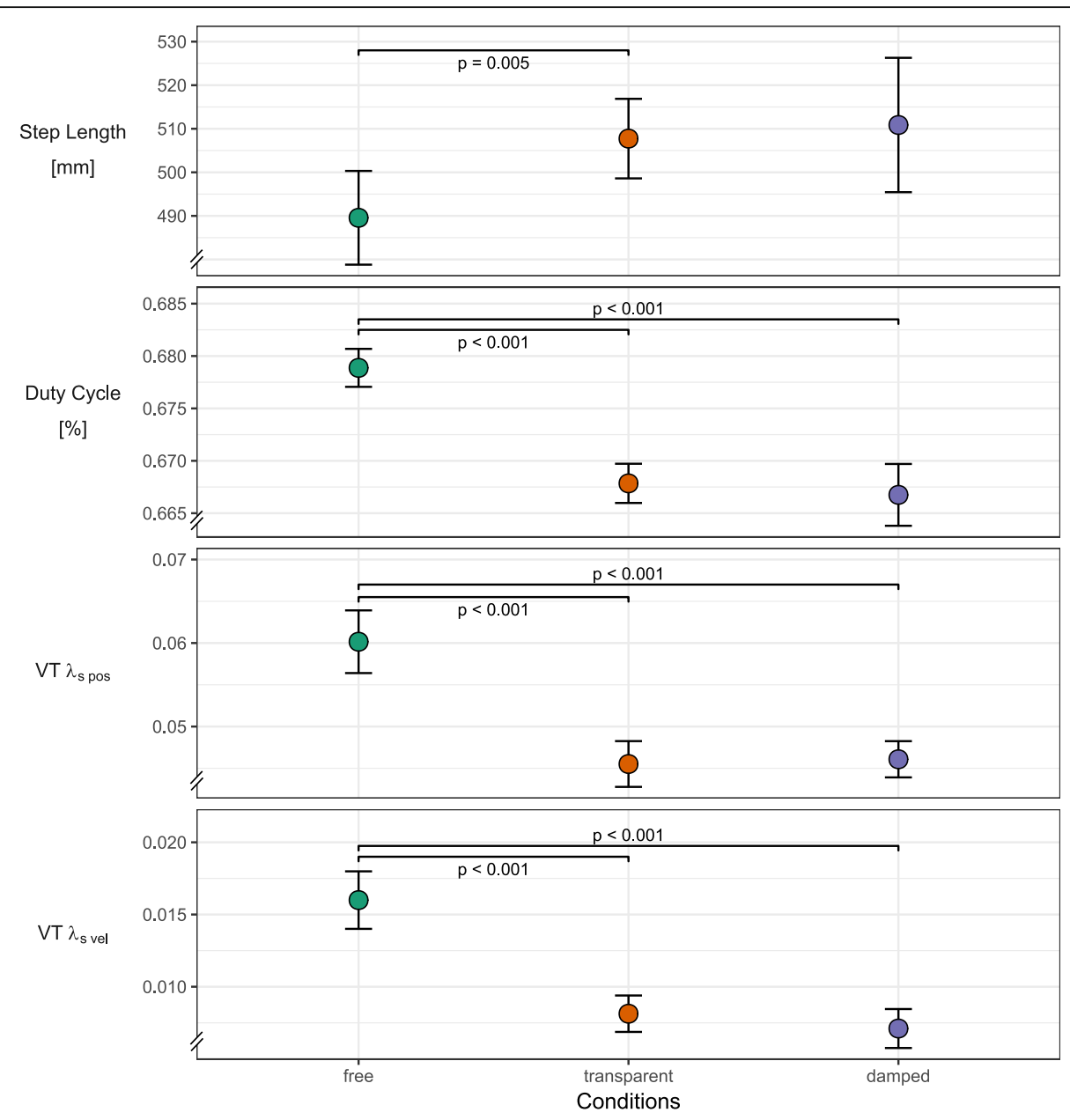

Fig. 3 Effects of damped and undamped BWS on the anteroposterior and vertical axes. Line plots of estimated marginal means over the three stability conditions for outcomes related to the anteroposterior and vertical axes. Plot includes error bars specifying 95\%-confidence intervals (within-subject, [71]) and statistically significant differences denoted by $p$-values. Abbreviations: VT - vertical, $\lambda_{s}$ pos - short-term maximum Lyapunov exponent calculated from approximated center of mass position, $\lambda_{s}$ vel - short-term maximum Lyapunov exponent calculated from approximated center of mass velocity

\section{Discussion}

In this study, we investigated the use of ML damping with a 3-DoF BWS system to support dynamic stability in ablebodied individuals walking on a treadmill. Participants walked in 3 different conditions: freely without BWS system attached, with $30 \%$ BWS but in a transparent device mode, and with 30\% BWS and ML velocity-based damping. Our results show that damping indeed increases frontal plane dynamic stability compared to transparent and free walking conditions. This effect is mainly visible in kinematic variability and ML local dynamic parameters, while direct global dynamic stability parameters show no coherent response. Systematic changes of direct global dynamic stability parameters may be masked by compensatory movements aimed at accentuating active weight transfer. Adapting the damping level individually to the abilities of the participants in future studies might however reduce such compensatory effects.

\section{Body weight support effects}

Literature on how BWS affects gait patterns of individuals both with $[3,27]$ and without neurological impairments $[3,26]$ indicates that small adaptations abound without grossly distorting either movement or myoelectric patterns. Our study results are in agreement with this. Additionally, we show that besides spatiotemporal parameters, dynamic stability is also clearly affected by BWS. From the selected outcome parameters, only the MoS were not decreased by BWS. Parameters which were equally affected in transparent and damped conditions were duty cycle, step length and local dynamic stability measured by $\lambda_{s}$ along the VT axis. These parameters reacted primarily in response to the applied $30 \%$ BWS in both conditions. The decrease in duty cycle along with the subsequent increase in step length [27, $72,73]$ has been frequently reported with regard to unloading. One explanation is that cadence decreases 


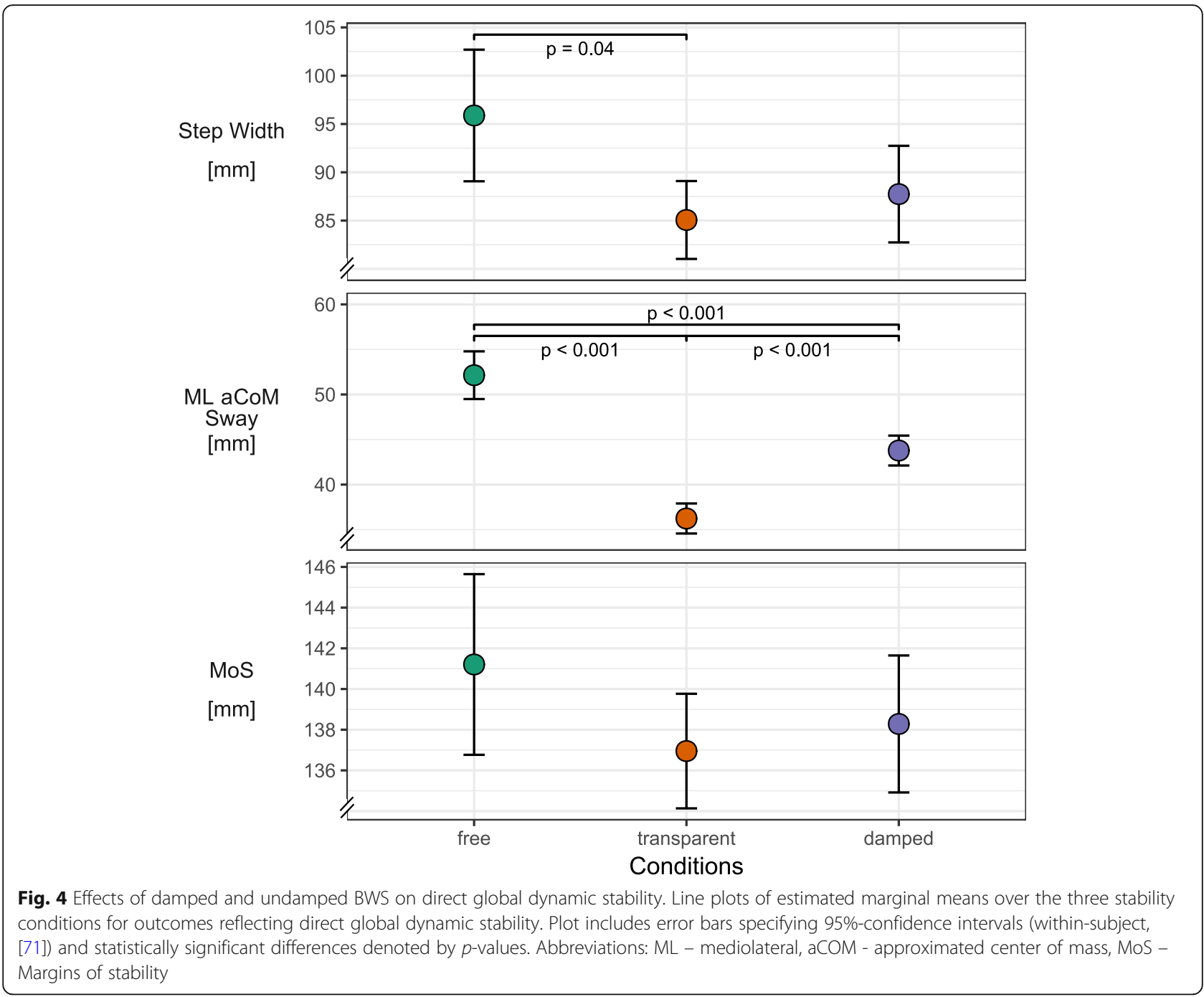

with BWS [26] which must be compensated by longer steps to maintain the same walking velocity [27] given by the fixed treadmill speed. With the reduced cadence, step duration becomes larger which is attributed to the influence of a prolonged swing phase while the stance phase remains less affected [27]. This change in relative swing and stance phase duration is reflected in the reduced duty cycle [21, 72-76]. An increase in local dynamic stability, as quantified by $\lambda_{s}$ of the aCOM, has on the other hand not yet been reported in combination with body weight support conditions. Intuitively, it seems reasonable that unloading per se acts as a damper and reduces peak velocities, especially in earthward direction. This is reflected in the reduction of VT $\lambda_{s}$ pos and VT $\lambda_{\mathrm{s} \text { vel. }}$ BWS also reduces ML $\lambda_{\mathrm{s} \text { pos }}$ and ML $\lambda_{\mathrm{s}}$ vel, indicating greater local dynamic stability in the frontal plane than during free walking. Variability of step width [58] along with ML aCOM sway and its variability were also reduced, which is in line with the general belief that BWS per se already increases dynamic stability [72, 77, 78]. Along the sagittal plane, Kyvelidou et al. found however increased $\lambda_{\mathrm{s}}$ and kinematic variability of the hip, knee, and ankle angles during walking with BWS on a treadmill [79]. They hypothesize that BWS might increase balance demands along the AP direction or that the instability is an effect of altered proprioception due to reduced limb loading. We did not measure dynamic stability based on lower limb joints, so there is the possibility that in both studies trunk stability increased while lower limb stability decreased. The relationship of the different contributors to stability is an important aspect to keep in mind when BWS is used to train patients with balance impairments and will require further investigation in the future.

There are other factors related to sensory perception which might contribute to the observed dynamic stability improvements. The linkage between harness and end-effector provides subjects with haptic feedback of 


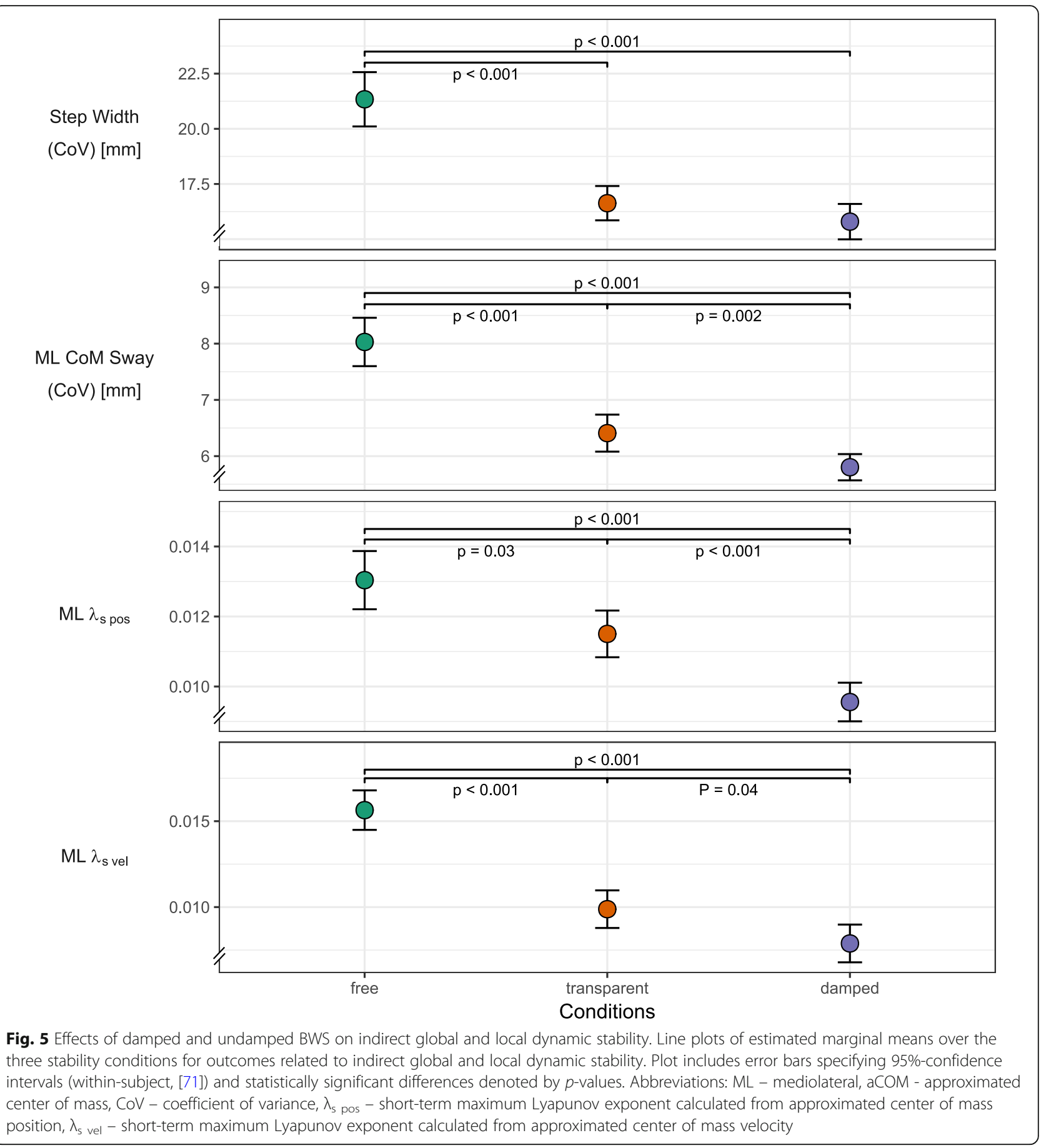

their own position in relation to the end-effector position. This might aid subjects in sensing their own lateral position on the treadmill and may result in a less variable ML walking position [36]. Changes in global dynamic stability have been reported when subjects are allowed to lightly touch a side rail while walking [80, 81]. Light touch resulted in decreased ML COM sway and ML COM sway variability on a treadmill and ML MoS variability during overground walking, respectively.
It is currently unclear if light touch would also affect local dynamic stability measured by $\lambda_{s}$. Further studies are necessary to disentangle how much of the here observed dynamic stability gains can be attributed to BWS effects or stem from improved sensory perception.

\section{Effects of damping}

To our best knowledge, our investigation of how ML damping of the end-effector affects dynamic stability 
during walking with BWS is completely novel. Our results indicate that adding ML damping on top of BWS provides enhanced dynamic stability. This is visible from reductions in global and local stability parameters between the free and transparent as well as between the transparent and damped conditions. Specifically, step width variability, ML aCOM sway variability and ML $\lambda_{s}$ pos and $\lambda_{\mathrm{s}}$ vel were affected in a graded manner. Apart from step width variability for the transparent versus damped condition, all reductions were highly significant. Step width variability is often considered to reflect balance demands [34]. It increases for instance, when the eyes are closed $[34,54]$ or when visual perturbations are presented [82]. Multiple studies have previously investigated the sensitivity of step width variability to changed balance demands. These studies report increased variability in older adults [52, 83], decreased variability when walking with handrail usage [52], and a persistent decrease in variability with external stabilization through elastic springs [40-44]. Destabilizing force fields which increased the balance demands have been shown to increase step width variability [84]. Other studies have reported conflicting results and relate decreased step width variability to sensory impairments and fall risk [53]. Additionally, studies investigating attention during walking have also shown reduced step width variability under additional cognitive load [85]. The same was found for ML COM variability in a subsequent study and it was hypothesized that individuals adopt a more conservative gait pattern when attention is diverted from foot placement [86]. These studies would posit decreased variability of step width and ML COM as proxies of reduced stability. We therefore consider change in variability alone as not conclusive in differentiating between reduced or increased ML dynamic stability. $\lambda_{s}$ on the other hand has proven to be more robust regarding the direction of change and can distinguish well between different stability demands $[60,87,88]$. As both kinematic variability and ML $\lambda_{\mathrm{s}}$ in our study show the same response, we consider this a robust indication that damping indeed improves dynamic stability in ablebodied participants.

Time-invariant parameters traditionally associated with global dynamic stability - such as step width [89], ML aCOM sway, and MoS [60, 90] - do however not seem to reflect the increased stability indicated by reduced variability and $\lambda_{s}$ when damping is added. One potential explanation for this is that we did not perturb the system sufficiently. Variability and $\lambda_{\mathrm{s}}$ are thought to represent the system's resilience to small perturbations as would be induced through the added ML damping. Global dynamic stability, on the other hand, represents the system's resilience to large perturbations. These were potentially not encountered in our experimental conditions, explaining the lack of a cohesive response pattern in these parameters. A second potential explanation could be that the aCOM motion is exaggerated compared to the base of support to compensate for the ML damping. Exaggerated ML aCOM motion could help participants to retain a target, or "preferred", step width. Veneman et al. have reported similar effects for half of their subjects when investigating pelvis fixations for a driven gait orthosis [91]. In our experiment, the BWS harness could well have restricted trunk excursion to some degree. Restricted trunk excursion has been reported to be linked with matched changes in step width $[89,92]$, giving indication that exaggerated trunk excursion could indeed help in maintaining a target step width. In summary, direct metrics of global dynamic stability show no coherent response to damping, presumably as the perturbation was insufficiently large or compensatory aCOM movement masked the response. The observed reductions in variability parameters and ML local dynamic stability coherently indicate an increase in stability when damping is applied.

\section{Clinical implications}

From the current investigation, it is clear that damping provides a stabilizing effect in able-bodied individuals. The stabilization we showed here could be helpful for gait and balance training in patients with balance impairments. It is however still unclear how large this stabilizing effect is and if it provides enough stabilization to support patients with balance impairments. If the damping provides sufficient stabilization, training can be started at an earlier time point in rehabilitation when self-balance capacity is still limited. Using BWS in combination with a stabilization adapted to the patient's needs, critical training parameters such as walking distance and training intensity can be increased. Both the early start of gait training and increase in training intensity has been shown to result in better recovery [93-95]. Finding the optimal balance for frontal plane stability assistance for each patient remains crucial, as overuse of stability support can result in patients slacking and becoming passive which decreases recovery [96]. An elegant aspect of using control-rendered damping is that the amount of stabilization can be tailored to each patient's individual instantaneous level. This enables training at the patient's optimal threshold while avoiding frequent and therefore time-consuming falls. The alternative option of adding handheld assistive devices to the training is in contradiction with established locomotor training principles [32, 97] as well as with emerging principles of reinforcing functional remapping through arm use $[10,11,14]$.

From a handful of gait trainings of patients with incomplete spinal cord injury with a damped BWS end-effector, 
we can report that patients and their therapists subjectively perceive damping as an assistance which increases stability. A previous investigation by $\mathrm{Wu}$ et al. in which ML damping was provided without BWS directly to the pelvis of patients with incomplete spinal cord injury showed a reduction in step width and increased MoS [84]. This provides conceptual support that ML damping at the endeffector level can lead to improved dynamic stability in patients. Future investigations are needed to uncover the exact magnitude of stabilization that ML damping provides in clinical populations. In contrast to able-bodied subjects, psychological effects must be considered when measuring global and local stability in patients. In non-fallers, an increased fear of falling has been associated with higher $\lambda_{s}$ [98]. In some groups of patients, dynamic stability could be increased alone through the perception of BWS as a safety aid. These and other design considerations are paramount when evaluating the approach of software-rendered viscous damping at the end-effector level as a stability aid for BWS locomotor training in clinical populations.

\section{Limitations}

Three main limitations were identified in this study. First, COM kinematics were approximated through the intersection of the four pelvis markers. This has been reported to differ from gold standard COM calculations based on a full-body marker set especially along the ML axis under high walking speeds [99]. Under slower walking speeds comparable to ours, multiple studies have however shown that approximating the COM results in accurate COM estimations along the $\mathrm{AP}, \mathrm{ML}$, and VT directions [100-102]. The reduced marker set was chosen as even our reduced harness obscured trunk marker placements. Normal BWS harnesses make marker placements at both trunk and pelvis challenging, which renders the use of a full-body marker set for COM calculation questionable in this setting. Second, we described subjects' motions through kinematics alone, which does not allow conclusions about how neuromuscular control was adapted in response to the external stabilization. Electromyography of the muscles involved in ML stability control along with modelling of the non-trivial human-robot system would be necessary to answer such questions which was beyond the scope of this study. Finally, it is important to keep in mind that the here obtained results can unfortunately not be generalized directly to overground walking. We decided to measure stability changes resulting from ML damping on a treadmill, as the nonlinear dynamic modeling approach requires a large amount of continuous walking data that cannot easily be recorded overground [103]. The necessary long measurement durations of around 10 min might also have led to learning or adaptation effects [84]. We counteracted this by introducing significant washout and familiarization periods before each measurement to limit carry-over effects to subsequent conditions.

\section{Conclusion}

In this study, we provide first insights into the effect of ML damping in 3-DoF BWS systems on walking stability. We demonstrate that adding viscous damping during body weight supported treadmill walking increases local dynamic stability and attenuates movement variability in able-bodied subjects. This damped BWS mode can be used to support patients with balance impairments during locomotor training in a 3-DoF BWS system without introducing additional stability aids. This form of providing a stability aid is very elegant, as it allows easy, continuous adjustment of the damping to each patient's instantaneous capacity. In ablebodied subjects, we however also observed what we believe to be compensatory aCOM movements to counteract movement limitations of the end-effector when the system was damped. This underlines the importance of tailoring the amount of support to each patient's capacity to achieve an optimum between stability support and challenge during training. Further studies are necessary to show how patient groups react to different levels of mediolateral damping of the BWS end-effector in terms of walking stability.

\section{Abbreviations \\ $\lambda_{s}$ : Short-term maximum Lyapunov exponent; aCOM: Approximated center of mass; AP: Anteroposterior; BWS: Body weight support; BoS: Base of support; COM: Center of mass; DoF: Degrees of freedom; ML: Mediolateral; MoS: Margins of stability; RM-MANOVA: Repeated measures multivariate analysis of variance; $V \mathrm{~T}$ : Vertical}

\section{Acknowledgements}

We thank Dr. Lea Awai for her feedback and editing suggestions. Furthermore, we would like to thank all subjects who volunteered to participate in this study.

\section{Authors' contributions}

M.Ba., S.B., and C.E. performed the measurements. M.Ba., G.R., M.B. and C.E. were involved in planning and supervised the work. M.Ba., S.B. and C.E. processed the experimental data and contributed together with N.KI. to the analysis. M.Ba. and C.E. drafted the manuscript and designed the figures. All authors were involved in the study design, discussed the results,

commented and edited the manuscript. The final manuscript was read and approved by all authors

\section{Funding}

This project was funded by Innosuisse (formerly the Commission of Technology and Innovation), Grant number 17567.2 PFLS-LS and Balgrist Campus AG (Swiss Center for Clinical Movement Analysis, SCMA).

\section{Availability of data and materials}

The datasets used and/or analyzed during the current study are available from the corresponding author on reasonable request.

Ethics approval and consent to participate

Participants gave written informed consent prior to participating in this study, which was approved by the local ethics committee of the Canton of Zurich (KEK Nr. PB 2016-0193) and was conducted in accordance with the Declaration of Helsinki. 


\section{Consent for publication}

Not applicable.

\section{Competing interests}

The authors declare that they have no competing interests.

\section{Author details}

'Spinal Cord Injury Center, Balgrist University Hospital, University of Zurich, Zurich, Switzerland. ${ }^{2}$ Sensory Motor Systems Laboratory, Department of Health Sciences and Technology, Swiss Federal Institute of Technology, Zurich, Switzerland. ${ }^{3}$ Department of Physical Therapy, Chapman University, Irvine, USA. ${ }^{4}$ BIROMED-Laboratory, Department of Biomedical Engineering, University of Basel, Basel, Switzerland. ${ }^{5}$ cereneo Center for Interdisciplinary Research, Vitznau, Switzerland.

\section{Received: 23 January 2020 Accepted: 28 July 2020}

\section{Published online: 10 August 2020}

\section{References}

1. Feigin VL, Nichols E, Alam T, Bannick MS, Beghi E, Blake N, et al. Global, regional, and national burden of neurological disorders, 1990-2016: a systematic analysis for the global burden of disease study 2016. Lancet Neurol. 2019;18:459-80.

2. Stolze $H$, Klebe $S$, Baecker C, Zechlin C, Friege L, Pohle S, et al. Prevalence of gait disorders in hospitalized neurological patients. Mov Disord. 2005;20:8994.

3. Apte $\mathrm{S}$, Plooij $\mathrm{M}$, Vallery $\mathrm{H}$. Influence of body weight unloading on human gait characteristics: a systematic review. J Neuroeng Rehabil. 2018;15:53.

4. Harkema SJ, Hillyer J, Schmidt-Read M, Ardolino E, Sisto SA, Behrman AL. Locomotor Training: As a treatment of spinal cord injury and in the progression of neurologic rehabilitation. Arch Phys Med Rehabil. 2012;93: 1588-97 Elsevier Inc.

5. Winter D. Human balance and posture control during standing and walking. Gait Posture. 1995;3:193-214.

6. Park J. Synthesis of natural arm swing motion in human bipedal walking. J Biomech. 2008:41:1417-26.

7. Wu Y, Li Y, Liu A-M, Xiao F, Wang Y-Z, Hu F, et al. Effect of active arm swing to local dynamic stability during walking. Hum Mov Sci. 2016;45:102-9.

8. Meyns P, Bruijn SM, Duysens J. The how and why of arm swing during human walking. Gait Posture. 2013;38:555-62 Elsevier B.V.

9. Goudriaan M, Jonkers I, van Dieen JH, Bruijn SM. Arm swing in human walking: What is their drive? Gait Posture. 2014;40:321-6 Elsevier B.V.

10. Kaupp C, Pearcey GEP, Klarner T, Sun Y, Cullen H, Barss TS, et al. Rhythmic arm cycling training improves walking and neurophysiological integrity in chronic stroke: the arms can give legs a helping hand in rehabilitation. J Neurophysiol. 2018;119:1095-112.

11. Klimstra MD, Thomas E, Stoloff RH, Ferris DP, Zehr EP. Neuromechanical considerations for incorporating rhythmic arm movement in the rehabilitation of walking. Chaos An Interdiscip J Nonlinear Sci. 2009;19: 026102.

12. Sylos-Labini F, Ivanenko YP, MacLellan MJ, Cappellini G, Poppele RE, Lacquaniti F. Locomotor-Like Leg Movements Evoked by Rhythmic Arm Movements in Humans. PLoS One. 2014;9:e90775 van Beers RJ, editor.

13. Zhou R, Alvarado L, Ogilvie R, Chong SL, Shaw O, Mushahwar VK. Non-gaitspecific intervention for the rehabilitation of walking after SCl: role of the arms. J Neurophysiol. 2018;119:2194-211.

14. Balter JE, Zehr EP. Neural coupling between the arms and legs during rhythmic Locomotor-like cycling movement. J Neurophysiol. 2007:97:1809-18.

15. Sim YJ, Lee DR, Yi CH, Cynn HS. Effects of repetitive intensive arm swing indirect gait training on vasti and hamstring muscle activity and gait performance in children with cerebral palsy. Int J Ther Rehabil. 2020;0:1-13.

16. Buurke TJW, Lamoth CJC, van der Woude LHV, den Otter R. Handrail holding during treadmill walking reduces Locomotor learning in ablebodied persons. IEEE Trans Neural Syst Rehabil Eng. 2019;27:1753-9 IEEE

17. Proietti T, Crocher V, Roby-Brami A, Jarrasse N. Upper-limb robotic exoskeletons for Neurorehabilitation: a review on control strategies. IEEE Rev Biomed Eng. 2016;9:4-14

18. Frey M, Colombo G, Vaglio M, Bucher R, Jorg M, Riener R. A novel mechatronic body weight support system. IEEE Trans Neural Syst Rehabil Eng. 2006;14:311-21.
19. Hesse S, Waldner A, Tomelleri C. Innovative gait robot for the repetitive practice of floor walking and stair climbing up and down in stroke patients. J Neuroeng Rehabil. 2010;7:30.

20. Brown DA, Lee TD, Reinkensmeyer DJ, Duarte JE. Designing robots that challenge to optimize motor learning. In: Reinkensmeyer DJ, Dietz V, editors. Neurorehabilitation Technology. 2nd ed. Cham: Springer International Publishing; 2016. p. 39-58.

21. Fischer AG, Wolf A. Assessment of the effects of body weight unloading on overground gait biomechanical parameters. Clin Biomech. 2015;30:454-61 Elsevier Ltd.

22. Fabara E, O'Brien A, Vergara-Diaz G, Adans-Dester C, Bonato P. Usability of a new over-ground bodyweight support device (Andago ${ }^{\circledR} 2.0$ ) for gait training. Arch Phys Med Rehabil. 2016;97:e134.

23. Hidler J, Brennan D, Black I, Nichols D, Brady K, Nef T. ZeroG: Overground gait and balance training system. J Rehabil Res Dev. 2011;48:287.

24. Vallery H, Lutz P, von Zitzewitz J, Rauter G, Fritschi M, Everarts C, et al. Multidirectional transparent support for overground gait training. In: 2013 IEEE 13th Int Conf Rehabil Robot. Seattle: IEEE; 2013. p. 1-7.

25. Plooij M, Keller U, Sterke B, Komi S, Vallery H, von Zitzewitz J. Design of RYSEN: an intrinsically safe and low-power three-dimensional Overground body weight support. IEEE Robot Autom Lett. 2018;3:2253-60.

26. Awai L, Franz M, Easthope CS, Vallery H, Curt A, Bolliger M. Preserved gait kinematics during controlled body unloading. J Neuroeng Rehabil. 2017;14:25.

27. Easthope CS, Traini LR, Awai L, Franz M, Rauter G, Curt A, et al. Overground walking patterns after chronic incomplete spinal cord injury show distinct response patterns to unloading. J Neuroeng Rehabil. 2018;15:102.

28. Bannwart M, Rohland E, Easthope CA, Rauter G, Bolliger M. Robotic body weight support enables safe stair negotiation in compliance with basic locomotor principles. J Neuroeng Rehabil. 2019;16:157.

29. Stolze H, Klebe S, Zechlin C, Baecker C, Friege L, Deuschl G. Falls in frequent neurological diseases. J Neurol. 2004;251:79-84.

30. Lubetzky-Vilnai A, Kartin D. The effect of balance training on balance performance in individuals Poststroke. J Neurol Phys Ther. 2010;34:127-37.

31. Harkema SJ, Schmidt-Read M, Lorenz DJ, Edgerton VR, Behrman AL. Balance and Ambulation Improvements in Individuals With Chronic Incomplete Spinal Cord Injury Using Locomotor Training-Based Rehabilitation. Arch Phys Med Rehabil. 2012;93:1508-17 Elsevier Inc.

32. Spiess MR, Steenbrink F, Esquenazi A. Getting the best out of advanced rehabilitation Technology for the Lower Limbs: minding motor learning principles. PM\&R. 2018;10:S165-73.

33. Barbeau H. Locomotor training in Neurorehabilitation: emerging rehabilitation concepts. Neurorehabil Neural Repair. 2003:17:3-11.

34. Bauby CE, Kuo AD. Active control of lateral balance in human walking. J Biomech. 2000;33:1433-40.

35. Meyer G, Ayalon M. Biomechanical aspects of dynamic stability. Eur Rev Aging Phys Act. 2006;3:29-33.

36. Dragunas AC, Gordon KE. Body weight support impacts lateral stability during treadmill walking. J Biomech. 2016;49:2662-8 Elsevier.

37. Fischer AG, Wolf A. Body weight unloading modifications on frontal plane joint moments, impulses and Center of Pressure during overground gait. Clin Biomech. 2016;39:77-83 Elsevier Ltd.

38. Pennycott A, Wyss D, Vallery H, Riener R. Effects of added inertia and body weight support on lateral balance control during walking. In: 2011 IEEE Int Conf Rehabil Robot. Zurich: IEEE; 2011. p. 1-5.

39. Wyss D, Bartenbach V, Pennycott A, Riener $R$, Vallery H. A body weight support system extension to control lateral forces: Realization and validation. In: 2014 IEEE Int Conf Robot Autom. Hong Kong: IEEE; 2014. p. 328-332.

40. Donelan JM, Shipman DW, Kram R, Kuo AD. Mechanical and metabolic requirements for active lateral stabilization in human walking. J Biomech. 2004:37:827-35.

41. Dean JC, Alexander NB, Kuo AD. The effect of lateral stabilization on walking in Young and old adults. IEEE Trans Biomed Eng. 2007:54:1919-26.

42. ljmker T, Houdijk H, Lamoth CJC, Beek PJ, van der Woude LHV. Energy cost of balance control during walking decreases with external stabilizer stiffness independent of walking speed. J Biomech. 2013;46:2109-14 Elsevier.

43. Mahaki M, Bruijn SM, Van Dieen J. The effect of external lateral stabilization on the use of foot placement to control mediolateral stability in walking and running. PeerJ. 2019;7:e7939.

44. Matsubara JH, Wu M, Gordon KE. Metabolic cost of lateral stabilization during walking in people with incomplete spinal cord injury. Gait Posture. 2015;41:646-51. 
45. Frame HB. The influence of lateral stabilization on walking performance and balance control in healthy and post-stroke individuals [dissertation on the Internet]. Austin; University of Texas at Austin; 2018 [cited 2019 Dec 15]. Available from: https://repositories.lib.utexas.edu/handle/2152/72456.

46. van Emmerik REA, Ducharme SW, Amado AC, Hamill J. Comparing dynamical systems concepts and techniques for biomechanical analysis. J Sport Heal Sci. 2016;5:3-13 Elsevier B.V.

47. Meyer C, Killeen T, Easthope CS, Curt A, Bolliger M, Linnebank M, et al. Familiarization with treadmill walking: how much is enough? Sci Rep. 2019; 9:5232.

48. Owings TM, Grabiner MD. Measuring step kinematic variability on an instrumented treadmill: how many steps are enough? J Biomech. 2003:36:1215-8.

49. Kang $\mathrm{HG}$, Dingwell JB. Intra-session reliability of local dynamic stability of walking. Gait Posture. 2006;24:386-90.

50. Bannwart M, Bolliger M, Lutz P, Gantner M, Rauter G. Systematic Analysis of Transparency in the Gait Rehabilitation Device The FLOAT. In: 14th International Conference on Control, Automation, Robotics and Vision (ICARCV); Phuket: IEEE; 2016. p. 1-6.

51. Havens KL, Mukherjee T, Finley JM. Analysis of biases in dynamic margins of stability introduced by the use of simplified center of mass estimates during walking and turning. Gait Posture. 2018;59:162-7.

52. Owings TM, Grabiner MD. Variability of step kinematics in young and older adults. Gait Posture. 2004;20:26-9

53. Brach JS, Berlin JE, VanSwearingen JM, Newman AB, Studenski SA. Too much or too little step width variability is associated with a fall history in older persons who walk at or near normal gait speed. J Neuroeng Rehabil. 2005;2:21.

54. Collins SH, Kuo AD. Two Independent Contributions to Step Variability during Over-Ground Human Walking. PLoS One. 2013;8:e73597 Balasubramaniam $\mathrm{R}$, editor.

55. Riva F, Bisi MC, Stagni R. Orbital stability analysis in biomechanics: A systematic review of a nonlinear technique to detect instability of motor tasks. Gait Posture. 2013;37:1-11 Elsevier B.V.

56. Hausdorff JM. Gait variability: methods, modeling and meaning. J Neuroeng Rehabil. 2005;2:19.

57. Hamacher D, Singh NB, Van Dieen JH, Heller MO, Taylor WR. Kinematic measures for assessing gait stability in elderly individuals: a systematic review. J R Soc Interface. 2011;8:1682-98.

58. König Ignasiak N, Ravi DK, Orter S, Hosseini SH, Taylor WR, Singh NB, et al. Does variability of footfall kinematics correlate with dynamic stability of the Centre of mass during walking? Anson ER, editor. PLoS One. 2019;14:1-14 Public Library of Science.

59. Rispens SM, Pijnappels M, van Dieën JH, van Schooten KS, Beek PJ, Daffertshofe A. A benchmark test of accuracy and precision in estimating dynamical systems characteristics from a time series. J Biomech. 2014:47:470-5 Elsevier.

60. Bruijn SM, Meijer OG, Beek PJ, van Dieën JH. Assessing the stability of human locomotion: a review of current measures. J R Soc Interface. 2013;10:20120999.

61. Zeni JA, Richards JG, Higginson JS. Two simple methods for determining gait events during treadmill and overground walking using kinematic data. Gait Posture. 2008;27:710-4.

62. Stergiou N. Nonlinear analysis for human movement variability. Nonlinear anal. Hum. Mov. Var. Boca Raton: CRC Press; 2016.

63. Takens F. Detecting strange attractors in turbulence; 1981. p. 366-81.

64. Stine RA, Abarbanel HDI. Analysis of observed chaotic data. Technometrics. 1997;39:334

65. Buzug T, Pfister G. Optimal delay time and embedding dimension for delaytime coordinates by analysis of the global static and local dynamical behavior of strange attractors. Phys Rev A. 1992;45:7073-84.

66. Kennel MB, Brown R, Abarbanel HDI. Determining embedding dimension for phase-space reconstruction using a geometrical construction. Phys Rev A. 1992;45:3403-11.

67. Fraser AM, Swinney HL. Independent coordinates for strange attractors from mutual information. Phys Rev A. 1986;33:1134-40.

68. Wolf A, Swift JB, Swinney HL, Vastano JA. Determining Lyapunov exponents from a time series. Physica. 1985;16D:285-317.

69. Korkmaz S, Goksuluk D, Zararsiz G. MVN: an R package for assessing multivariate normality. R J. 2014;6:151

70. Tabachnick BG, Fidell LS. Using Multivariate Statistics. 7th edition. New York: Pearson; 2019.

71. Morey RD. Confidence intervals from normalized data: a correction to Cousineau (2005). Tutor Quant Methods Psychol. 2008;4:61-4.
72. Threlkeld AJ, Cooper LD, Monger BP, Craven AN, Haupt HG. Temporospatial and kinematic gait alterations during treadmill walking with body weight suspension. Gait Posture. 2003;17:235-45.

73. van Hedel HJA, Tomatis L, Müller R. Modulation of leg muscle activity and gait kinematics by walking speed and bodyweight unloading. Gait Posture. 2006;24:35-45.

74. Ruckstuhl H, Kho J, Weed M, Wilkinson MW, Hargens AR. Comparing two devices of suspended treadmill walking by varying body unloading and Froude number. Gait Posture. 2009;30:446-51.

75. Donelan JM, Kram R. The effect of reduced gravity on the kinematics of human walking: a test of the dynamic similarity hypothesis for locomotion. J Exp Biol. 1997;200:3193-201.

76. Sylos-Labini F, Ivanenko YP, Cappellini G, Portone A, MacLellan MJ, Lacquaniti F. Changes of gait kinematics in different simulators of reduced gravity. J Mot Behav. 2013:45:495-505.

77. Sousa CO, Barela JA, Prado-Medeiros CL, Salvini TF, Barela AM. The use of body weight support on ground level: an alternative strategy for gait training of individuals with stroke. J Neuroeng Rehabil. 2009;6:43.

78. Finch L, Barbeau H, Arsenault B. Influence of body weight support on Normal human gait: development of a gait retraining strategy. Phys Ther. 1991;71:842-55.

79. Kyvelidou A, Kurz MJ, Ehlers JL, Stergiou N. Aging and partial body weight support affects gait variability. J Neuroeng Rehabil. 2008:5:22.

80. Dickstein $R$, Laufer $Y$. Light touch and center of mass stability during treadmill locomotion. Gait Posture. 2004;20:41-7.

81. Oates A, Unger J, Arnold C, Fung J, Lanovaz J. The effect of light touch on balance control during overground walking in healthy young adults. Heliyon. 2017;3:e00484 Elsevier Ltd.

82. O'Connor SM, Xu HZ, Kuo AD. Energetic cost of walking with increased step variability. Gait Posture. 2012;36:102-7 Elsevier B.V.

83. Skiadopoulos A, Moore EE, Sayles HR, Schmid KK, Stergiou N. Step width variability as a discriminator of age-related gait changes. J Neuroeng Rehabil J Neuroeng Rehabil. 2020;17:41.

84. Wu M, Brown G, Gordon KE. Control of locomotor stability in stabilizing and destabilizing environments. Gait Posture. 2017;55:191-8.

85. Grabiner MD, Troy KL. Attention demanding tasks during treadmill walking reduce step width variability in young adults. J Neuroeng Rehabil. 2005;2:25.

86. Dingwell JB, Robb RT, Troy KL, Grabiner MD. Effects of an attention demanding task on dynamic stability during treadmill walking. J Neuroeng Rehabil. 2008:5:12.

87. Dingwell JB, Marin LC. Kinematic variability and local dynamic stability of upper body motions when walking at different speeds. J Biomech. 2006;39: 444-52.

88. Toebes MJP, Hoozemans MJM, Furrer R, Dekker J, van Dieën JH. Local dynamic stability and variability of gait are associated with fall history in elderly subjects. Gait Posture. 2012;36:527-31.

89. Bruijn SM, van Dieën JH. Control of human gait stability through foot placement. J R Soc Interface. 2018;15:20170816.

90. Hof AL. The 'extrapolated center of mass' concept suggests a simple control of balance in walking. Hum Mov Sci. 2008;27:112-25.

91. Veneman JF, Menger J, van Asseldonk EHF, van der Helm FCT, van der Kooij $H$. Fixating the pelvis in the horizontal plane affects gait characteristics. Gait Posture. 2008;28:157-63.

92. Arvin M, van Dieën JH, Bruijn SM. Effects of constrained trunk movement on frontal plane gait kinematics. J Biomech. 2016:49:3085-9 Elsevier.

93. Nudo RJ. Recovery after brain injury: mechanisms and principles. Front Hum Neurosci. 2013;7:887.

94. Bernhardt J, Godecke E, Johnson L, Langhorne P. Early rehabilitation after stroke. Curr Opin Neurol. 2017;30:48-54.

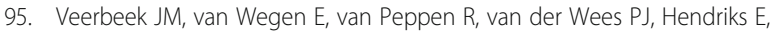
Rietberg $\mathrm{M}$, et al. What is the evidence for physical therapy Poststroke? A Systematic Review and Meta-Analysis. PLoS One. 2014;9:e87987.

96. Wolbrecht ET, Chan V, Reinkensmeyer DJ, Bobrow JE. Optimizing compliant, model-based robotic assistance to promote Neurorehabilitation. IEEE Trans Neural Syst Rehabil Eng. 2008;16:286-97.

97. Harkema S, Behrman A, Barbeau H. Evidence-based therapy for recovery of function after spinal cord injury. Handb Clin Neurol. 2012;109:259-74 1st ed. Elsevier B.V.

98. Toebes MJP, Hoozemans MJM, Furrer R, Dekker J, van Dieën JH. Associations between measures of gait stability, leg strength and fear of falling. Gait Posture. 2015:41:76-80. 
99. Süptitz F, Catalá MM, Brüggemann G-P, Karamanidis K. Dynamic stability control during perturbed walking can be assessed by a reduced kinematic model across the adult female lifespan. Hum Mov Sci. 2013;32:1404-14 Elsevier B.V.

100. McAndrew Young PM, Wilken JM, Dingwell JB. Dynamic margins of stability during human walking in destabilizing environments. J Biomech. 2012;45: 1053-9 Elsevier.

101. Whittle MW. Three-dimensional motion of the center of gravity of the body during walking. Hum Mov Sci. 1997;16:347-55.

102. Gard SA, Miff SC, Kuo AD. Comparison of kinematic and kinetic methods for computing the vertical motion of the body center of mass during walking. Hum Mov Sci. 2004;22:597-610.

103. Orter S, Ravi DK, Singh NB, Vogl F, Taylor WR, König IN. A method to concatenate multiple short time series for evaluating dynamic behaviour during walking. PLoS One. 2019;14:1-14.

\section{Publisher's Note}

Springer Nature remains neutral with regard to jurisdictional claims in published maps and institutional affiliations.

Ready to submit your research? Choose BMC and benefit from:

- fast, convenient online submission

- thorough peer review by experienced researchers in your field

- rapid publication on acceptance

- support for research data, including large and complex data types

- gold Open Access which fosters wider collaboration and increased citations

- maximum visibility for your research: over $100 \mathrm{M}$ website views per year

At $\mathrm{BMC}$, research is always in progress.

Learn more biomedcentral.com/submissions 